\title{
ON $(0,1,2)$ INTERPOLATION IN UNIFORM METRIC
}

\author{
J. SZABADOS AND A. K. VARMA
}

(Communicated by R. Daniel Mauldin)

\begin{abstract}
From the well known theorem of G. Faber it follows that for any given matrix of nodes there exists a continuous function for which the Lagrange interpolation polynomial $L_{n}[f, x]$, generated by the $n$th row of the matrix, does not tend uniformly to $f(x)$. In this paper we shall provide analogous results for the related operator $H_{n, 3}[f, x]$ as defined below.
\end{abstract}

Let

$$
(-1 \leq) x_{1}<x_{2}<\cdots<x_{n}(\leq 1)
$$

be an arbitrary system of nodes of interpolation $\left(x_{k}=x_{k n}, k=1, \ldots, n ; n=\right.$ $1,2, \ldots)$, and for an arbitrary continuous function $f(x)$ in $[-1,1]$ (i.e., $f \in C[1,1])$ and integer $m \geq 1$, consider the $(0,1, \ldots, m)$ Hermite-Fejér interpolation of order $m$ defined by

$$
H_{n, m}^{(j)}\left(f, x_{k}\right)=\delta_{0, j} f\left(x_{k}\right)(k=1, \ldots, n ; j=0, \ldots, m-1) .
$$

Evidently, $H_{n, m}(f, x)$ is a uniquely determined polynomial of degree at most $m n-1$.

$H_{n, 1}(f, x)$ is the Lagrange interpolation polynomial of $f(x)$; the classical result of $\mathrm{G}$. Faber [4] shows that this cannot be uniformly convergent for all $f \in C[-1,1]$ for any system of nodes (1); while another classical result of $\mathrm{P}$. Erdös and P. Turán [2] asserts that if (1) are the roots of the $n$th orthogonal polynomial with respect to an arbitrary $L^{1}$-integrable weight function $w(x) \geq$ 0 , then $H_{n, 1}(f, x)$ converges in weighted $L^{2}$ metric for any $f \in C[-1,1]$.

For $m=2$, the situation is different. There exist systems of nodes (1) such that $H_{n, 2}(f, x)$ uniformly converges for all $f \in C[-1,1]$ (e.g., for the roots of the Jacobi polynomials $P_{n}^{(\alpha, \beta)}(x)$ with $-1<\alpha, \beta<0$; see G. Szegö [6], Theorem 14.6). Hence in this case the $L^{2}$ convergence follows automatically and is of no interest.

Received by the editors December 1, 1988; this paper was presented by the second author at the Sixth Texas International Symposium on Approximation Theory, January 1989.

1980 Mathematics Subject Classification (1985 Revision). Primary 41 A05.

This paper was completed while the first author visited the University of Florida in Gainesville. Research partially supported by Hungarian National Foundation for Scientific Research Grant No. 1801 . 
For $m=3$ the results are less complete. R. Sakai [5] proved that for the Chebyshev roots $H_{n, 3}(f, x)$ cannot converge for all $f \in C[-1,1]$ (actually he proved this for all odd $m$ 's), and later P. Vértesi [7, Theorem 2.7] generalized this result for arbitrary Jacobi nodes (under some additional condition whose validity is checked only for $m \leq 5)$. Our result here is that for any system of nodes (1), $H_{n, 3}(f, x)$ cannot converge uniformly for all $f \in C[-1,1]$. This follows from the following more quantitative result on the norm of $H_{n, 3}$.

Theorem. For any system of nodes (1) we have

$$
|| H_{n, 3} \mid \| \geq c \log n .
$$

Proof. An easy calculation shows that

$$
H_{n, 3}(f, x)=\sum_{k=1}^{n} f\left(x_{k}\right) A_{k}(x)
$$

where

$$
\begin{array}{r}
A_{k}(x)=\left\{1-3 l_{k}^{\prime}\left(x_{k}\right)\left(x-x_{k}\right)+\left[6 l_{k}^{\prime}\left(x_{k}\right)^{2}-\frac{3}{2} l_{k}^{\prime \prime}\left(x_{k}\right)\right]\left(x-x_{k}\right)^{2}\right\} l_{k}(x)^{3} \\
(k=1, \ldots, n)
\end{array}
$$

with the usual notation $l_{k}(x)$ for the $k$ th fundamental polynomial of Lagrange interpolation. Hence

$$
\left\|H _ { n , 3 } \left|\left\|\left|=\left\|\sum_{k=1}^{n}\left|A_{k}(x)\right|\right\|\right.\right.\right.\right.
$$

where $\|\cdot\|$ is the supremum norm of the corresponding function. Since 1 $3 x+4.5 x^{2}>0$ for any real $x$, we get from (4)

$$
1-3 l_{k}^{\prime}\left(x_{k}\right)\left(x-x_{k}\right)+\left[6 l_{k}^{\prime}\left(x_{k}\right)^{2}-\frac{3}{2} l_{k}^{\prime \prime}\left(x_{k}\right)\right]\left(x-x_{k}\right)^{2} \geq \frac{3}{2}\left[l_{k}^{\prime}\left(x_{k}\right)^{2}-l_{k}^{\prime \prime}\left(x_{k}\right)\right]\left(x-x_{k}\right)^{2} \text {. }
$$

Here

$l_{k}^{\prime}\left(x_{k}\right)^{2}-l_{k}^{\prime \prime}\left(x_{k}\right)=\left(\sum_{i \neq k} \frac{1}{x_{k}-x_{i}}\right)^{2}-\sum_{\substack{i, j \neq k \\ i \neq j}} \frac{1}{\left(x_{k}-x_{i}\right)\left(x_{k}-x_{j}\right)}=\sum_{i \neq k} \frac{1}{\left(x_{k}-x_{i}\right)^{2}}$, i.e.,

$$
\left|A_{k}(x)\right| \geq \frac{3}{2} \frac{\left(x-x_{k}\right)^{2}}{\left(x_{k}-x_{k-1}\right)^{2}}\left|l_{k}(x)\right|^{3} \quad(2 \leq k \leq n) .
$$

In the rest of the proof of Theorem 1 we use a modification of the idea of proof of Theorem 1 in P. Erdös and P. Turán [3]. We distinguish two cases.

Case 1. There is a $k_{0}, 1 \leq k_{0} \leq n$ such that

$$
\left|l_{k_{0}}\left(\xi_{0}\right)\right|=\left\|l_{k_{0}}\right\| \geq n^{2} \quad\left(\xi_{0} \in[-1,1]\right) .
$$


Then by Markov's theorem

$$
\left|l_{k_{0}}(x)\right| \geq \frac{1}{2}\left\|l_{k_{0}}\right\| \geq \frac{1}{2} n^{2} \quad\left(\left|x-\xi_{0}\right| \leq \frac{1}{2 n^{2}}\right) .
$$

Thus choosing a $\xi_{1} \in[-1,1]$ such that $\left|\xi_{1}-x_{k_{0}}\right| \geq \frac{1}{2 n^{2}} \geq\left|\xi_{1}-\xi_{0}\right|$ we obtain from (5) and (6)

$$
\left|A_{k_{0}}\left(\xi_{1}\right)\right| \geq \frac{3}{2} \cdot \frac{1}{4} \cdot\left(\frac{1}{2 n^{2}}\right)^{2}\left(\frac{1}{2} n^{2}\right)^{3}=\frac{3}{2^{8}} n^{2},
$$

which is stronger than (2).

Case 2. $\left\|l_{k}\right\|<n^{2}(k=1,2, \ldots, n)$. In this case, according to a result of $\mathrm{P}$. Erdös [1], we have (with $x_{k}=\cos \theta_{k}$ )

$$
\left|\sum_{\theta_{k} \in I} 1-\frac{|I|}{\pi} n\right| \leq \log ^{2} n \quad(I \subseteq[0, \pi])
$$

Thus

$$
\sum_{\theta_{k} \in I} 1 \geq \frac{1}{15}|I| n \text { if }|I| \geq 4 \frac{\log ^{2} n}{n}, I \subseteq[0, \pi] \text { and } n \geq n_{0} .
$$

Therefore using the harmonic-geometric-arithmetic mean inequalities we get

$$
\begin{aligned}
\sum_{\theta_{k} \in I} \frac{1}{\left(x_{k}-x_{k-1}\right)^{2}} & \geq \frac{\sum_{\theta_{k} \in I} 1}{\left(\prod_{\theta_{k} \in I}\left(\theta_{k-1}-\theta_{k}\right)\right)^{2 / \sum_{\theta_{k} \in I}}} \geq \frac{\left(\sum_{\theta_{k} \in I} 1\right)^{3}}{\left[\sum_{\theta_{k} \in I}\left(\theta_{k-1}-\theta_{k}\right)\right]^{2}} \\
& \geq \frac{\left(\frac{1}{15}|I| n\right)^{3}}{|I|^{2}}>10^{-4}|I| n^{3} \text { if }|I| \geq 4 \frac{\log ^{2} n}{n}, I \subseteq[0, \pi], n \geq n_{0} .
\end{aligned}
$$

Now let

(8) $\omega_{n}(x)=\prod_{k=1}^{n}\left(x-x_{k}\right), I_{n}=\left[-\frac{1}{\log n}, \frac{1}{\log n}\right], I_{n}^{\prime}=\left[-\frac{1}{2 \log n}, \frac{1}{2 \log n}\right]$,

(9) $M_{n}=\max _{|x| \leq 1}\left|\omega_{n}(x)\right|=\left|\omega_{n}(\xi)\right|, \bar{M}_{n}=\max _{x \in I_{n}}\left|\omega_{n}(x)\right|=\left|\omega_{n}\left(\xi_{0}\right)\right| \quad(x=\cos \theta)$.

According to Lemma 1 in [3], we have

$$
\max _{\theta \in I_{n}^{\prime}}\left|\omega_{n}^{\prime}(x)\right|=O\left(\eta_{n} n M_{n}\right), \text { where } \eta_{n}=\max \left(\frac{1}{\log ^{2} n}, \frac{\bar{M}_{n}}{M_{n}}\right) \text {. }
$$

Subcase 2 a. $\bar{M}_{n} \leq M_{n} / \log ^{2} n$. Then by (10)

$$
\max _{\theta \in I_{n}^{\prime}}\left|\omega_{n}^{\prime}(x)\right|=O\left(\frac{n M_{n}}{\log ^{2} n}\right) \text {. }
$$


Choosing $I=I_{n}^{\prime}$ in (7), we obtain from (5), (8), (9), and (7)

$$
\begin{aligned}
\sum_{\theta_{k} \in I_{n}^{\prime}}\left|A_{k}(\xi)\right| & \geq \frac{3}{4} \sum_{\theta_{k} \in I_{n}^{\prime}}\left|\frac{\omega_{n}(\xi)}{\omega_{n}^{\prime}\left(x_{k}\right)}\right|^{3} \frac{1}{\left(x_{k}-x_{k-1}\right)^{2}} \\
& \geq c_{1} \frac{\log ^{6} n}{n^{3}} \sum_{\theta_{k} \in I_{n}^{\prime}} \frac{1}{\left(x_{k}-x_{k-1}\right)^{2}} \geq c_{2} \log ^{5} n .
\end{aligned}
$$

Subcase $2 \mathrm{~b} . \bar{M}_{n}>M_{n} / \log ^{2} n$. Then similarly as above,

$$
\max _{. \theta \in I_{n}^{\prime}}\left|\omega_{n}^{\prime}(x)\right|=O\left(n \bar{M}_{n}\right),
$$

and

$$
\begin{aligned}
\sum_{\theta_{k} \in I_{n}^{\prime}}\left|A_{k}\left(\xi_{0}\right)\right| & \geq \frac{3}{2} \sum_{\theta_{k} \in I_{n}^{\prime}}\left|\frac{\omega_{n}\left(\xi_{0}\right)}{\omega_{n}^{\prime}\left(x_{k}\right)}\right|^{3} \frac{1}{\left|\xi_{0}-x_{k}\right|\left(x_{k}-x_{k-1}\right)^{2}} \\
& \geq \frac{c_{3}}{n^{3}} \sum_{\theta_{k} \in I_{n}^{\prime}} \frac{1}{\left|\xi_{0}-x_{k}\right|\left(x_{k}-x_{k-1}\right)^{2}} .
\end{aligned}
$$

Let $\xi_{0}=\cos \theta_{0}$ and $0<\theta_{0} \leq \frac{\pi}{2}$. Define

$$
\begin{aligned}
I_{n, \lambda}=\left[\theta_{0}+4 \lambda \frac{\log ^{2} n}{n}, \theta_{0}+4(\lambda+1) \frac{\log ^{2} n}{n}\right] \subseteq I_{n}^{\prime} \\
\left(\lambda=0,1, \ldots,\left[\frac{n}{10 \log ^{3} n}\right]=m\right) .
\end{aligned}
$$

Then by (7) (with $I=I_{n, \lambda}$ )

$$
\begin{aligned}
\sum_{\theta_{k} \in I_{n}^{\prime}} \frac{1}{\left|\xi_{0}-x_{k}\right|\left(x_{k}-x_{k-1}\right)^{2}} & \geq \sum_{\lambda=1}^{m} \sum_{\theta_{k} \in I_{n, \lambda}} \frac{1}{\left|\theta_{0}-\theta_{k}\right|\left(x_{k}-x_{k-1}\right)^{2}} \\
& \geq c_{4} \frac{n}{\log ^{2} n} \sum_{\lambda=1}^{m} \frac{1}{\lambda} \sum_{\theta_{k} \in I_{n, \lambda}} \frac{1}{\left(x_{k}-x_{k-1}\right)^{2}} \\
& \geq c_{5} \frac{n}{\log ^{2} n} \cdot \log n \cdot \log ^{2} n \cdot n^{2} \\
& =c_{5} n^{3} \log n,
\end{aligned}
$$

i.e., by (11)

$$
\sum_{\theta_{k} \in I_{n}^{\prime}}\left|A_{k}\left(\xi_{0}\right)\right| \geq c_{3} c_{5} \log n
$$

The theorem is completely proved.

Remark. We conjecture that the statement of our theorem remains true for any odd $m$. 


\section{REFERENCES}

1. P. Erdös, On the uniform distribution of the roots of certain polynomials, Ann. of Math. $\mathbf{4 3}$ (1942), 59-64.

2. P. Erdös and P. Turán, On interpolation. I, Ann. of Math. 38 (1937), 142-155.

3. An extremal problem in the theory of interpolation, Acta Math. Acad. Sci. Hung. 12 (1961), 221-233.

4. G. Faber, Über die interpolatorische Darstellung stetiger Funktionen, Jahresber. der Deutschen Math. Ver. 23 (1914), 190-210.

5. R. Sakai, Hermite-Fejér interpolation prescribing higher order derivatives, J. Approx. Theory (to appear).

6. G. Szegö, Orthogonal Polynomials, vol. 23, Amer. Math. Soc. Colloq. Publ., Providence, RI, 1974.

7. P. Vértesi, Hermite-Fejér interpolations of higher order. I, Acta Math. Hungarian Academy, (1-2) 59 (1989), 135-152.

Mathematical Institute of the Hungarian Academy of Sciences, Budapest, ReáltanODA U.13-15 H-1053

University of Florida, Department of Mathematics, Gainesville, Florida 32611 